\title{
Dynamical response of an excitatory-inhibitory neural network to external stimulation: An application to image segmentation
}

\author{
Sitabhra Sinha ${ }^{1,2}$ and Jayanta Basak ${ }^{3, *}$ \\ ${ }^{1}$ Department of Physics, Indian Institute of Science, Bangalore 560 012, India \\ ${ }^{2}$ Condensed Matter Theory Unit, Jawaharlal Nehru Center for Advanced Scientific Research, Bangalore 560 064, India \\ ${ }^{3}$ Machine Intelligence Unit, Indian Statistical Institute, Calcutta 700 035, India
}

\begin{abstract}
Neural network models comprising elements that have exclusively excitatory or inhibitory synapses are capable of a wide range of dynamical behavior, including chaos. In this paper, a simple excitatory-inhibitory neural pair, which forms the building block of larger networks, is subjected to external stimulation. The response shows transition between various types of dynamics, depending upon the magnitude of the stimulus. The corresponding network model, obtained by coupling such pairs over a local neighborhood in a twodimensional plane, can achieve a satisfactory segmentation of an image into "object" and "background." Results for synthetic and "real-life" images are given.
\end{abstract}

\section{INTRODUCTION}

Dynamical transitions in brain activity, in the presence of an external stimulus, have received considerable attention recently. Most investigations of these phenomena have focused on the phase synchronization of oscillatory activity in neural assemblies. An example is the detection of synchronization of " $40-\mathrm{Hz}$ " oscillations within and between visual areas and between cerebral hemispheres of cats [1] and other animals. Assemblies of neurons have been observed to form and separate depending on the stimulus.

Schuster and Wagner $[2,3]$ have demonstrated the activity dependent coupling between the neuronal oscillators. The oscillators are tightly coupled in their active state and weakly coupled in their passive state, and the activity dependent couplings are independent of the underlying connectivities in the neural assemblies. Theoretical investigations are also made about the interactions between the weakly coupled assemblies of neuronal oscillators by Grannan et al. [4].

These studies have led to the speculation that phase synchronization of oscillatory neural activity is one of the key mechanisms for "visual binding." This is the process by which local stimulus features of an object (e.g., color, motion, shape), after being processed in parallel by different (spatially separate) regions of the cortex, are correctly integrated in higher brain areas, forming a coherent representation ("gestalt").

Sensory segmentation, the ability to pick out certain objects by segregating them from their surroundings, is a prime example of "binding." The problem of segmentation of sensory input is of primary importance in several fields. In the case of visual perception, "object-background" discrimination is the most obvious form of such sensory segmentation: the object to be attended to, is segregated from the surrounding objects in the visual field. Several methods for segmen-

\footnotetext{
*Present address: IBM India Research Lab, Block-I, IIT Campus, Hauz Khas, New Delhi 110 016, India
}

tation, both classical and connectionist, are reported in the literature [5].

Most of the studies on segmentation through neural assembly formation have concentrated on networks of oscillators that synchronize when representing the same object. Malsburg and co-workers [6] have sought to explain segmentation through dynamic link architecture with synapses that rapidly switch their functional state. Similar approaches using synaptic couplings that change rapidly depending on the stimulus have been used in a neural model for segmentation by Sporns et al. [7]. Grossberg and Sommers [8] have performed figure-ground separation with a network of oscillators, some of which belong to the "object" and the others to the "background." Oscillations of the former are synchronized, whereas the others have nonoscillatory activity. Han et al [9] have used an oscillatory network for Hopfield-type autoassociation in pattern segmentation, using the temporal dynamics of the nonlinear oscillators driven by noise and subthreshold periodic forcing. If the input is a superposition of several overlapping stored patterns, the network segments out each pattern successively, as synchronous activation of a group of "neurons." Similar segmentation through synchronization of activity among a cluster of neurons have been shown by other groups [10-14]. In an alternative approach, Sompolinsky and Tsodkys [15] have demonstrated the effectiveness of coherent coupling in feature segmentation. Several image patterns are stored in the model and the couplings are tuned by Hebb-like learning rule.

In contrast to these approaches, we present a method of utilizing the transition between different types of dynamics (e.g., between fixed-point and periodic behaviors) of the network elements, for performing segmentation tasks. Here, we investigate the dynamical response of an excitatoryinhibitory neural network model evolving in discrete time, to external stimulation of constant intensity (in time), I. In Sec. II, we look at how the behavior of an individual element within the network changes with $I$. A theoretical analysis has been presented for the transition from period-2 cycles to fixed-point behavior for an isolated excitatory-inhibitory pair (i.e., not coupled to any other element) with an external input 


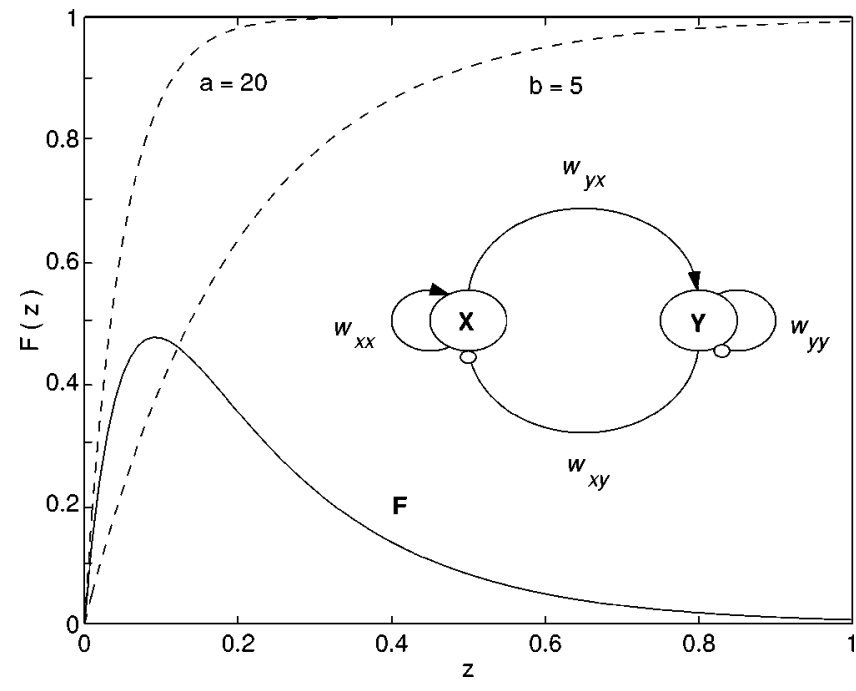

FIG. 1. The one-dimensional neural map, F [Eq. (4)]. The activation functions for the constituent excitatory (slope, $a=20$ ) and inhibitory $(b=5)$ neurons are also plotted using broken lines. The excitatory- $(x)$ inhibitory $(y)$ neural pair is shown as an inset. Links terminating in arrows indicate excitatory connections, while those terminating in circles indicate inhibitory connections.

of constant magnitude. The following section focuses on the role of lateral couplings in a network of such neural pairs. Simulation results for the spatially interacting coupled network are presented in Sec. IV, where the model is used to segment gray-level images. Finally, possible improvements of the proposed method are discussed in Sec. V.

\section{THE EXCITATORY-INHIBITORY NETWORK MODEL}

The model on which we have based our investigations comprises excitatory and inhibitory neurons, coupled to each other over a local neighborhood. As we are updating the model only at discrete time intervals, it is the time-averaged activity of the neurons that is being considered. Therefore, the "neuronal activity" can be represented as a continuous function, saturating to some maximum value, inversely related to the absolute refractory period. This is arbitrarily taken to be 1 , so that we can choose the following sigmoid function to be the neural activation function for our model:

$$
F_{\mu}(z)=\left\{\begin{array}{c}
1-\exp (-\mu z) \quad \text { if } \quad z \geqslant 0 \\
0 \quad \text { otherwise. }
\end{array}\right.
$$

The basic module of the proposed network is a pair of excitatory and inhibitory neurons coupled to each other (Fig. 1, inset). If $x$ and $y$ are the activities of the excitatory and the inhibitory elements, respectively, then they evolve in time according to

$$
\begin{aligned}
& x_{n+1}=F_{a}\left(w_{x x} x_{n}-w_{x y} y_{n}+I_{n}\right), \\
& y_{n+1}=F_{b}\left(w_{y x} x_{n}-w_{y y} y_{n}+I_{n}^{\prime}\right),
\end{aligned}
$$

where, $w_{i j}$ is the weight of synaptic coupling from element $j$ to element $i, F$ is the activation function defined by Eq. (1), and $I, I^{\prime}$ are external stimuli. By imposing the following restriction on the values of the synaptic weights: $w_{x y} / w_{x x}$ $=w_{y y} / w_{y x}=k$, and absorbing $w_{x x}$ and $w_{y y}$ within $a$ and $b$ (respectively), we can simplify the dynamics to that of the following one-dimensional difference equation or "map":

$$
z_{n+1}=F_{a}\left(z_{n}+I_{n}\right)-k F_{b}\left(z_{n}+I_{n}^{\prime}\right) .
$$

Without loss of generality, we can take $k=1$. In the following account we will be considering only time-invariant external stimuli, so that, for our purposes $I_{n}=I_{n}^{\prime}=I$. The resultant neural map exhibits a wide range of dynamics (fixed point, periodic, and chaotic), despite the simplicity of the model $[16,17]$.

\section{A. Dynamics of a single excitatory-inhibitory neural pair}

The autonomous behavior (i.e., $I, I^{\prime}=0$ ) of an isolated pair of excitatory-inhibitory neurons shows a transition from fixed point to periodic behavior and chaos with the variation of the parameters $a$ and $b$, following the "period-doubling" route, universal to all smooth, one-dimensional unimodal maps [18]. The map

$$
z_{n+1}=\mathbf{F}\left(z_{n}\right)=F_{a}\left(z_{n}\right)-F_{b}\left(z_{n}\right),
$$

describing the dynamics of the pair (Fig. 1), has two fixed points, at $z_{1}^{*}=0$ and $z_{2}^{*}$ [which is the solution of the transcendental equation $z=\exp (-b z)-\exp (-a z)]$. The fixed point $z_{1}^{*}$ is stable if the local slope $[\simeq(a-b)]$ is less than 1 . When this condition no longer holds, $z_{1}^{*}$ loses stability and $z_{2}^{*}$ becomes stable by a transcritical bifurcation. On further increase of $a$, this fixed point also loses stability and a period-2 cycle occurs. Increasing $a$ leads to a sequence of period doublings ultimately giving rise to chaos.

The introduction of an external stimulus of magnitude $I$ has the effect of horizontally displacing the map, Eq. (4), to the left by $I$. This implies that $z_{1}^{*}=0$ is no longer a fixed point, while the other fixed point $z_{2}^{*}$ is now a solution of the equation $z=\exp [-b(z+I)]-\exp [-a(z+I)]$. The slope at $z_{2}^{*}$ decreases with increasing $I$, giving rise to a reverse perioddoubling transition from chaos to periodic cycles to finally, fixed-point behavior.

\section{B. Analysis of response to constant magnitude external stimulus}

We shall now consider how the dynamics of the excitatory-inhibitory pair changes in response to external stimulus. The dynamics of the isolated neural pair is given by [using Eq.(1)]

$$
z_{n+1}=\exp \left[-b\left(z_{n}+I\right)\right]-\exp \left[-a\left(z_{n}+I\right)\right] .
$$

Note that, for $I>0$, only the stability of the nonzero fixed point $z_{2}^{*}$ needs to be examined. Hence, in what follows, $z^{*}$ $=z_{2}^{*}$. The fixed point is marginally stable if $(a-b) \exp$ $\left[-a\left(z^{*}+I_{c}\right)\right]=b z^{*}-1$, where, $I_{c}$ is the critical external stimu- 
lus for which $z^{*}$ just attains stability. Defining a new variable, $\alpha=\left(b z^{*}-1\right) /(a-b)$, the fixed point can be expressed as

$$
z^{*}=\frac{1}{b}+\frac{1-\mu}{\mu} \alpha
$$

where $\mu=b / a$, and the condition for marginal stability becomes

$$
\exp \left[-a\left(z^{*}+I_{c}\right)\right]=\alpha
$$

Now, from Eq. (5), the marginally stable fixed point can be expressed as $z^{*}=-\exp \left[-a\left(z^{*}+I_{c}\right)\right]+\exp \left[-b\left(z^{*}+I_{c}\right)\right]$. So, from Eqs. (6) and (7), we have

$$
\alpha=\frac{1}{b^{1 / \mu}}(1+a \alpha)^{1 / \mu}
$$

Assuming $a \alpha \ll 1$, only the first order terms in $\alpha$ need to be considered, so that

$$
\alpha=\frac{1}{b^{1 / \mu}-\frac{a}{\mu}} .
$$

For a real solution of $z^{*}$ to exist, we must have $\alpha>0$, i.e., $a<\mu b^{1 / \mu}$. Since $b=\mu a, a>\mu^{(\mu+1) /(\mu-1)}$. For example, if $\mu=0.5$, then $a>8$ for $z^{*}$ to be real. From the condition for marginal stability and Eq. (6), one obtains

$$
I_{c}=\left(\frac{\mu-1}{\mu}\right) \alpha-\frac{1}{\mu a}-\frac{1}{a} \ln (\alpha) .
$$

This equation, together with Eq. (9), provides the critical value of the external stimulus that drives the oscillatory neuron pair to a fixed stable state, subject to the restriction that $z^{*}$ is real.

This expression can be further simplified. From Eq. (8), one can write $\mu \ln (\alpha)=-\ln (b)+\ln (1+a \alpha)$. As before, assuming $a \alpha \ll 1$, we need to consider only the first order terms in $\alpha$ in the right hand side of the logarithmic expansion, so that

$$
\ln (\alpha)=\frac{a \alpha}{\mu}-\frac{1}{\mu} \ln (b) .
$$

From Eqs. (9), (10), and (11), the critical magnitude of the external stimulus can be expressed as

$$
I_{c}=\frac{1-\frac{2}{\mu}}{(\mu a)^{1 / \mu}-\frac{a}{\mu}}+\frac{1}{\mu a}[\ln (\mu a)-1] .
$$

Figure 2 shows the $a$ vs $I_{c}$ curves for different values of $\mu$, viz., $\mu=0.1,0.25$, and 0.5 .

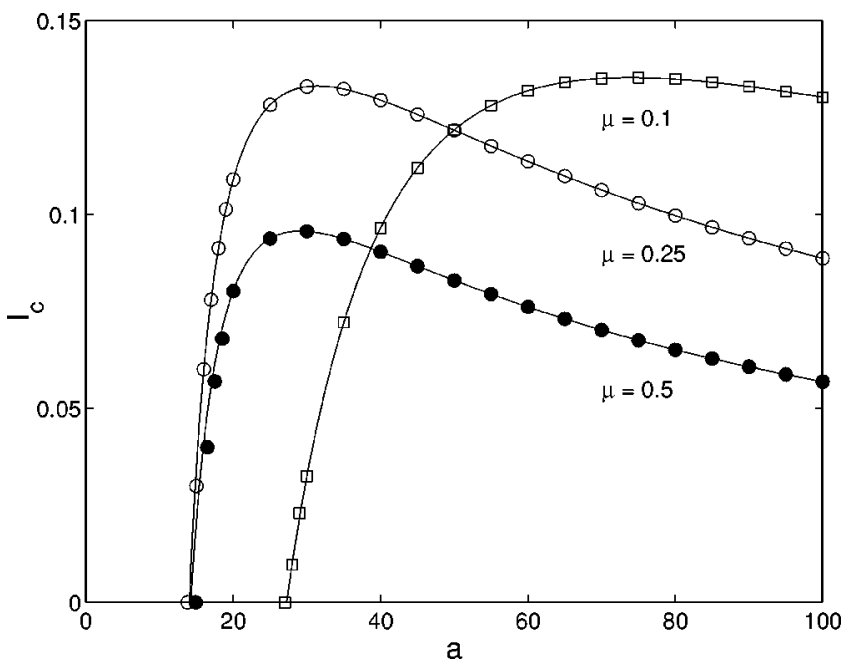

FIG. 2. Critical magnitude $\left(I_{c}\right)$ of the external stimulus, at which transition from periodic to fixed-point behavior occurs. The circles (filled and blank) and squares represent the values obtained through numerical procedures for $\mu=0.5,0.25$, and 0.1 , respectively. The curves indicate the theoretically predicted values obtained from Eq. (12).

\section{Choosing the operating region}

To make the network segment regions of different intensities $\left(I_{1}<I_{2}\right.$, say), one can fix $\mu$ and choose a suitable $a$, such that $I_{1}<I_{c}<I_{2}$. So elements, which receive input of intensity $I_{1}$, will undergo oscillatory behavior, while elements receiving input of intensity $I_{2}$, will go to a fixed-point solution. Notice that, the curves obtained from Eq. (12) give two values of $a$ for the same $I_{c}$. This gives rise to an operational question: given a certain $I_{c}$, which value of $a$ is more appropriate? Notice that, the region of the $a$ vs $I_{c}$ curve [Fig. (2)] to the left of the maxima, has a very high gradient. This implies that, in the presence of wide variation in the possible value of $I_{c}$, choice of $a$ from this region will show very small variation, i.e., the system performance will be robust with respect to uncertainty in the determination of the appropriate value of $I_{c}$. This is possible in the case of any graylevel image with a bimodal intensity distribution, having a long, almost uniform valley in between the two maxima.

On the other hand, the region of the curve to the right of the maxima has a very low gradient (almost approaching zero for high values of $a$ ). This implies structural stability in network performance, as wide variation in choice of $a$ will give almost identical results. So, choice of $a$ from this region is going to make the network performance stable against parametric variations. As both robustness against uncertain input and stability against parametric variations are highly desirable properties in network computation, a trade-off seems to be involved here. The nature of the task in hand is going to be the determining factor of which value of $a$ we should choose for a specific $I_{c}$.

\section{THE TWO-DIMENSIONAL NETWORK OF EXCITATORY-INHIBITORY NEURAL PAIRS}

The introduction of spatial interactions over a local neighborhood in the above model produces some qualitative 
changes in the response of the network to external stimulus. We have considered discrete approximations of circular neighborhoods [19] of radii $\mathcal{R}_{\text {ex }}$ and $\mathcal{R}_{i n}(\mathcal{R}=1,2)$ for excitatory and inhibitory elements, respectively, in our simulations.

Let us consider the case $\mathcal{R}_{e x}=\mathcal{R}_{i n}$. Then the autonomous dynamics of the coupled network is given by

$$
z_{n+1}=\mathbf{F}\left(z_{n}+\frac{1}{\left|\mathcal{R}_{e x}\right|} \sum_{i \in \mathcal{R}_{e x}} z_{n}^{i}\right)
$$

where $|\mathcal{R}|$ indicates the number of neurons within a neighborhood. To see how well the network performs segmentation, let an external input $I$ be applied to each element in the network. In addition to the local dynamics for a single element, we now also need to take into account the role of lateral coupling between neighboring excitatory-inhibitory neural pairs. The lateral coupling enables some degree of local smoothing of isolated discontinuities in the image to be achieved (thereby markedly improving the segmentation performance of the network). This can be seen from the following argument. Let us assume that the $i$ th neuronal pair is given an input $I$ while the pairs surrounding it are subject to an input $I^{\prime}\left(I<I_{c}<I^{\prime}\right)$. In the absence of lateral couplings the surrounding pairs converge to fixed-point attractors while the $i$ th pair undergoes a period- 2 cycle. To see how the dynamics of the $i$ th pair is altered by the interaction with neighboring pairs, we look at the time evolution of successive time difference of the activity of the $i$ th pair $z^{i}$,

$$
\begin{aligned}
\delta z_{n}^{i}= & z_{n+1}^{i}-z_{n}^{i} \\
= & \mathbf{F}\left(z_{n}^{i}+\frac{1}{\left|\mathcal{R}_{e x}\right|} \sum_{j \in \mathcal{R}_{e x}} z_{n}^{j}+I\right) \\
& -\mathbf{F}\left(z_{n-1}^{i}+\frac{1}{\left|\mathcal{R}_{e x}\right|} \sum_{j \in \mathcal{R}_{e x}} z_{n-1}^{j}+I\right) .
\end{aligned}
$$

Note that, the contribution from the neighboring elements (which have all converged to the fixed-point attractor) is $\left(1 /\left|\mathcal{R}_{e x}\right|\right) \Sigma_{j \in \mathcal{R}_{e x}} z_{n}^{j}=\left(1 /\left|\mathcal{R}_{e x}\right|\right) \Sigma_{j \in \mathcal{R}_{e x}} z_{n-1}^{j}=z^{*}$, say. Assuming $\left|z_{n}^{i}-z^{*}\right| \ll 1$ and considering only the first order term in the expansion of Eq. (13) about $z=2 z^{*}+I$, we obtain

$$
\delta z_{n}^{i}=\left(\frac{\partial \mathbf{F}}{\partial z}\right)_{z=2 z^{*+I}} \delta z_{n-1}^{i} .
$$

Provided $\left(I_{c}-I\right)<z^{*}, \quad-1<\left.(\partial \mathbf{F} / \partial z)\right|_{z=2 z^{*+I}}<0$, so that, $\delta z_{n}^{i} \rightarrow 0$ as $n \rightarrow \infty$. Therefore, $z^{i}$ converges to fixed point as a result of the lateral coupling with neighboring excitatoryinhibitory neural pairs, allowing the isolated discontinuity to be smoothed out and classified as part of its neighborhood during segmentation.

There is an important feature to consider about the relative sizes of the neighborhoods of the excitatory and inhibitory neurons, $\mathcal{R}_{e x}$ and $\mathcal{R}_{\text {in }}$, respectively. The autonomous dynamics of a coupled network is given by

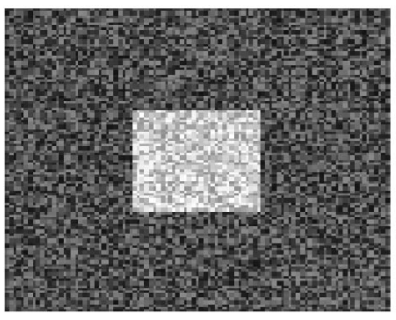

(I)

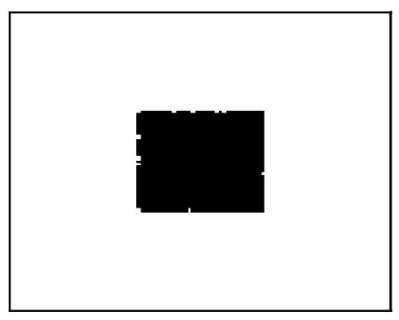

(c)

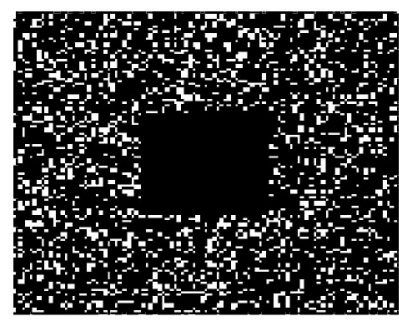

(b)

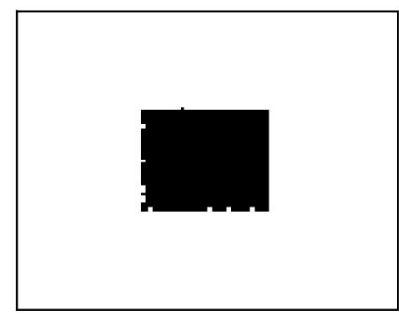

(d)
FIG. 3. Results of implementing the proposed segmentation method on a noisy synthetic image: (a) original image, (b) output of the uncoupled network, (c) output of the coupled network $\left(\mathcal{R}_{e x}\right.$ $\left.=1, \mathcal{R}_{i n}=2\right)$, and (d) output of the coupled network $\left(\mathcal{R}_{e x}=\mathcal{R}_{i n}\right.$ $=2$ ), after 200 iterations ( $a=20, \mu=0.25$ and threshold $\theta=0.02$ ).

$$
z_{n+1}=\mathbf{F}\left(z_{n}+\frac{1}{\left|\mathcal{R}_{e x}\right|} \sum_{i \in \mathcal{R}_{e x}} x_{n}^{i}-\frac{1}{\left|\mathcal{R}_{\text {in }}\right|} \sum_{i \in \mathcal{R}_{i n}} y_{n}^{i}\right) .
$$

This can be rewritten as

$$
\begin{aligned}
z_{n+1}= & \mathbf{F}\left(z_{n}+\frac{1}{\left|\mathcal{R}_{e x}\right|} \sum_{i \in \mathcal{R}_{e x}} z_{n}^{i}+\frac{1}{\left|\mathcal{R}_{e x}\right|} \sum_{i \in \mathcal{R}_{e x}} y_{n}^{i}\right. \\
& \left.-\frac{1}{\left|\mathcal{R}_{i n}\right|} \sum_{i \in \mathcal{R}_{i n}} y_{n}^{i}\right) .
\end{aligned}
$$

If $\mathcal{R}_{e x}=\mathcal{R}_{i n}$, then the equation reduces to

$$
z_{n+1}=\mathbf{F}\left(z_{n}+\frac{1}{\left|\mathcal{R}_{e x}\right|} \sum_{i \in \mathcal{R}_{e x}} z_{n}^{i}\right) .
$$

As $z_{n} \geqslant 0$ at all sites $i$, the activation increases with $n$, asymptotically driving the network to a homogeneous, uniformly activated state when $\mathcal{R}_{e x}=\mathcal{R}_{i n}$. However, for the times upto which we have simulated the network model, the output for the two cases $\mathcal{R}_{e x}=\mathcal{R}_{i n}$ and $\mathcal{R}_{e x}<\mathcal{R}_{i n}$ are qualitatively similar (as seen in Figs. 3 and 4).

\section{SIMULATION AND RESULTS}

The response behavior of the excitatory-inhibitory neural network with local couplings has been utilized in segmenting images and the results are shown in Figs. 3 and 4. Both synthetic and "real-life" gray-level images have been used. The initial state of the network is taken to be totally random. The image to be segmented is presented as external input to the network, which undergoes 200-300 iterations. Keeping $a$ fixed, a suitable value of $\mu$ is chosen from a consideration of the histogram of the intensity distribution of the image. This allows the choice of a value for the critical intensity $\left(I_{c}\right)$, such that, the neurons corresponding to the object converge to fixed-point behavior, while those belonging to the back- 


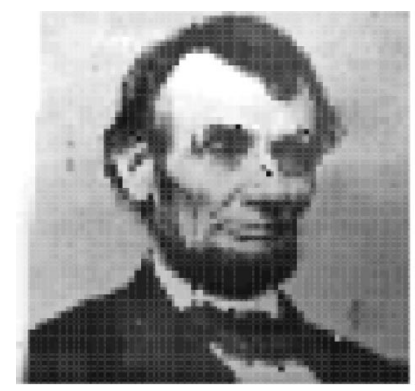

(a)

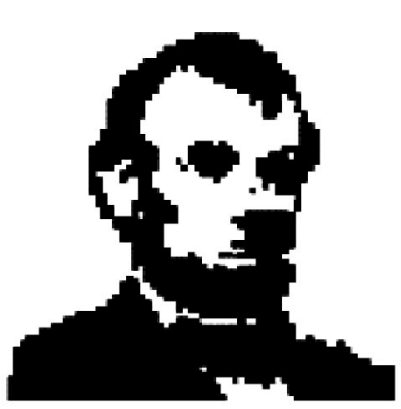

(c)

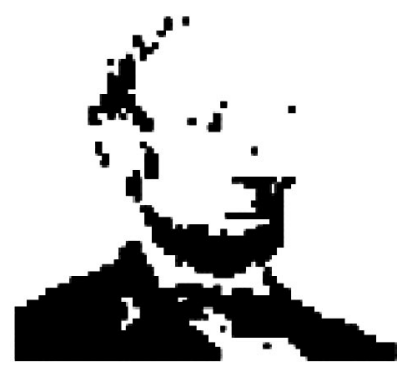

(b)

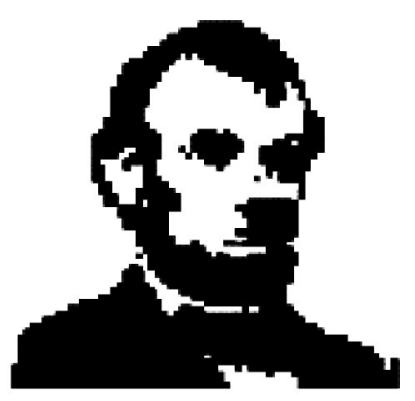

(d)
FIG. 4. Results of implementing the proposed segmentation method on "Lincoln" image: (a) original image, (b) output of the uncoupled network, (c) output of the coupled network $\left(\mathcal{R}_{e x}\right.$ $\left.=1, \mathcal{R}_{i n}=2\right)$, and (d) output of the coupled network $\left(\mathcal{R}_{e x}=\mathcal{R}_{i n}\right.$ $=2)$, after 300 iterations $(a=30, \mu=0.25$, and threshold $\theta$ $=0.02$ ).

ground undergo period- 2 cycles. In practice, after the termination of the specified number of iterations, the neurons that remain unchanged over successive iterations (within a tolerance value, $\theta$ ) are labeled as the object, the remaining being labeled the background.

The synthetic image chosen is that of a square of intensity $I_{2}$ (the object) against a background of intensity $I_{1}\left(I_{1}\right.$ $<I_{2}$ ). Uniform noise of intensity $\epsilon$ is added to this image. The signal-to-noise ratio (SNR) is defined as the ratio of the range of gray levels in the original image to the range of noise added (given by $\epsilon$ ). For $\mathrm{SNR}=1$, the results of segmentation are shown in Fig. 3. Figure 3(a) shows the original image while segmentation performance of the uncoupled network is presented in Fig. 3(b). As is clear from the figure, the isolated neurons perform poorly in identifying the background in the presence of noise. The segmentation performance improves remarkably when spatial interactions are included in the model. Results for $\mathcal{R}_{e x}=1, \mathcal{R}_{i n}=2$, and $\mathcal{R}_{\text {ex }}$ $=\mathcal{R}_{i n}=2$ are shown in Figs. 3(c) and 3(d), respectively. The two architectures show very similar segmentation results, at least upto the iterations considered here, although the latter is unstable (as discussed in the previous section). Excepting for the boundary of the object, which is somewhat broken, the rest of the image has been assigned to the two different classes quite accurately.

We have also considered the 5-bit gray level "Lincoln" image [Fig. 4(a)] as an example of a real-life picture. A suitable $I_{c}$ has been estimated by looking at the histogram of the gray-level values, and taking the trough between two dominating peaks as the required value. As in the synthetic image, the performance of a network of uncoupled neurons is not satisfactory [Fig. 4(b)]. The results of including spatial interaction are shown in Figs. 4(c) and 4(d). Most of the image has been labeled accurately, except for a few regions (e.g., near the neck).

Note that, we have considered a single value of $a$ (and hence $I_{c}$ ) for the entire image. This is akin to "global thresholding." By implementing local thresholding and choosing $a$ on the basis of local neighborhood information, the performance of the network can be improved.

\section{DISCUSSION}

In the present work, we have assumed constant connection weights over a local neighborhood. However, a Gaussian profile of weights may be biologically more realistic. One can also make the critical intensity $I_{c}$ proportional to the ambient intensity. This is in tune with how the retina seems to alter its sensitivity to incoming visual stimuli [20]. Finally, the role of variable connection weights, that can be implemented in the present model by changing the value of $k$ (ratio of the weights), may be investigated.

Chaotic neurodynamics has been used to segment images by Hasegawa et al. [21] and van Leeuwen et al. [22]. However, while the former approach is based on using chaos to avoid the local minima problem in the variable shape block segmentation method, the latter uses locally adaptive coupling to synchronize the chaotic activity of individual elements. We have instead concentrated on using stimulus induced transitions in neural network dynamics to segment images. As Malsburg [6] has indicated, the reason oscillatory synchronization has been studied so far as a mean of segmenting sensory stimuli is its relative ease of analysis. However, with the developments in nonlinear dynamics and chaos theory, we can advance to segmentation using more general dynamical behavior.

\section{ACKNOWLEDGMENTS}

We would like to thank Professor S. K. Pal (MIU, ISI, Calcutta) for his constant encouragement. S.S. acknowledges financial support from JNCASR.
[1] C.M. Gray, P. König, A.K. Engel, and W. Singer, Nature (London) 333, 334 (1989).

[2] H.G. Schuster and P. Wagner, Biol. Cybern. 64, 77 (1990).

[3] H.G. Schuster and P. Wagner, Biol. Cybern. 64, 83 (1990).

[4] E.R. Grannan, D. Kleinfeld, and H. Sompolinsky, Neural
Comput. 5, 550 (1993).

[5] R. C. Gonzalez and R. E. Woods, Digital Image Processing (Addison-Wesley, Reading, MA, 1992).

[6] C. von der Malsburg and J. Buhmann, Biol. Cybern. 67, 233 (1992). 
[7] O. Sporns, G. Tononi, and G.M. Edelman, Proc. Natl. Acad. Sci. U.S.A. 88, 129 (1991).

[8] S. Grossberg and D. Somers, Neural Networks 4, 453 (1991).

[9] S.K. Han, W.S. Kim, and H. Kook, Phys. Rev. E 58, 2325 (1998).

[10] H. Sompolinsky, D. Golomb, and D. Kleinfeld, Proc. Natl. Acad. Sci. U.S.A. 87, 7200 (1990).

[11] T.B. Schillen and P. König, Biol. Cybern. 70, 397 (1994).

[12] D.L. Wang, IEEE Trans. Neural Netw. 6, 941 (1995).

[13] S. Campbell and D.L. Wang, IEEE Trans. Neural Netw. 7, 541 (1996).

[14] H.J. Kappen, Phys. Rev. E 55, 5849 (1997).

[15] H. Sompolinsky and M. Tsodyks, Neural Comput. 6, 642
(1994).

[16] S. Sinha, Fundamenta Informaticae 37, 31 (1999).

[17] S. Sinha, Ph.D. thesis, Indian Statistical Institute, Calcutta, 1998.

[18] S. H. Strogatz, Nonlinear Dynamics and Chaos (AddisonWesley, Reading, MA, 1994).

[19] S.N. Biswas and B.B. Chaudhuri, Comput. Vis. Graph. Image Process. 32, 158 (1985).

[20] F.S. Werblin, Ann. N.Y. Acad. Sci. 190, 75 (1972).

[21] M. Hasegawa, T. Ikeguchi, T. Matozaki, and K. Aihara, IEICE Trans. Fundamentals 79, 1630 (1996).

[22] C. van Leeuwen, M. Steyvers, and M. Nooter, J. Math. Psychol. 41, 319 (1997). 